\title{
A Linearized Model of FID Signal for Increasing Proton Magnetometer Precision
}

\author{
Chao Tan ${ }^{1,2}$, Ruijie $\mathrm{Fu}^{1}$, Chenguang $\mathrm{Wu}^{1}$, Xinglin $\mathrm{Li}^{1}$ \\ ${ }^{I}$ College of Electrical Engineering \& New Energy, China Three Gorges University, Yichang 443002, China, \\ ctgutc@ctgu.edu.cn \\ ${ }^{2}$ Hubei Engineering Research Center of Weak Magnetic-field Detection, College of Science, China Three Gorges University, \\ Yichang 443002, China
}

\begin{abstract}
A linearized model of frequency measurement for the Free Induction Decay (FID) signal is proposed to increase the Proton Magnetometer (PM) precision. First, the nonlinear model of frequency measurement is set up according to the characteristic of the FID signal. Then, according to the error analysis of the MCFM method, the model is linearized on the condition of precision requirement. Furthermore, to reduce the nonlinear error caused by approximate treatment and the trigger time error caused by the random noise, the Least Squares (LS) method is adopted to estimate the slope of the linearized model, and the frequency to be measured is the inverse of the slope. Finally, a PM Prototype is made to verify the effectiveness of the proposed method. Experimental results show that the precision of frequency measurement is obviously increased if the proposed method is adopted for the noised sine signal. Moreover, the RMSD and the NPSD of magnetic-field measurement are about $0.13 \mathrm{nT}$ and $80 \mathrm{pT} / \mathrm{Hz}^{1 / 2}$, respectively if the proposed method is adopted by $\mathrm{PM}$, which is better than the comparison method.
\end{abstract}

Keywords: Proton Magnetometer, FID signal, frequency measurement, trigger time error, least square.

\section{INTRODUCTION}

The Proton Magnetometer (PM) is a weak magnetic-field measurement instrument based on the Nuclear Magnetic Resonance (NMR) of proton. The absolute precision of commercial PMs is about $\pm 0.5 \mathrm{nT}$, at present, it is up to $\pm 0.2 \mathrm{nT}$ due to the development of counting method and electronic technology [1]. So it has been widely used in the detection of static or quasistatic weak magnetic-fields, such as space exploration and geological exploration [2]-[4]. Nevertheless, the precision of commercial PMs is still not enough in some special applications such as magnetic-field metering, the absolute precision requirement of which is even smaller than $0.1 \mathrm{nT}$. The output signal of a PM sensor is called FID signal [5], [6], the frequency of the FID signal is proportional to the strength of the magnetic-field to be measured, and the magnitude of the FID signal is extremely weak - in the order of a few microvolts with an exponential decay, and is drowned by noise after approximately one second [7]. An amplified FID signal is displayed in Fig.1.a). As shown, the magnitude of the signal decays exponentially, and the envelope of the exponential curve displays large fluctuations, which indicate that the signal contains noise and the SNR is very low. The precision of PM is decided by the sensor, the amplifier circuit and the frequency measurement method [8]. If the sensor and the amplifier circuit have been fixed, the precision of the PM will be only decided by the frequency measurement precision of low SNR FID signal in a limited time frame. So, how to obtain accurate frequency values of low SNR FID signals is, therefore, key to improve the precision of PM. This makes research on high-precision frequency measurement methods in limited time frames, for low SNR sine signals, very important.

In the frequency domain method, the DFT method [9] is the most direct method for low SNR sine signal frequency estimation, and a lot of improved DFT methods [10]-[13] are developed to increase the precision. The frequency estimation precision can reach a high level if these aforementioned methods are adopted. However, a large number of samples and ADC sample rate are demanded. Furthermore, the feature of real-time will be decremented because of the complexity of algorithm. So, the application of these methods in portable devices will be limited. Counter method and its variations are the commonly used methods for frequency measurement [14], [15], which include the multi-cycle frequency measurement (MCFM) method, the discrete phase-delay detection method, and the frequency measurement method based on delay chains, and so on. The principle of these methods is that the sine signal to be measured is firstly 
converted to square wave at a comparator, and then the frequency of square wave is measured by the methods mentioned above. The precision of these methods is very high if there is no noise in signal, and method error is the main error for frequency measurement. However, for low SNR signal such as the FID signal, their performances will be decreased because trigger error caused by noise is far larger than method error. The IEEE-STD-1057 three- and fourparameter sine wave fit algorithms have been discussed in [16]-[19], the parameters of sine wave can be estimated accurately by curve fitting, but it is very time consuming because a lot of trigonometric operations must be executed.

There are some researchers focusing on how to increase the measurement precision of the FID signal. A Prony, SVD, and FDM method has been proposed in [20], [21], and [22], respectively, to measure the frequency of the FID signal, the simulations show that more than ten seconds are needed to obtain a high-precision result. A high-order Prony Method is presented in [23] to estimate the FID signal of NMR, but the time consumption is even higher than for the common Prony method. Dong [24] describes a delay multi-channel parallel measurement (MPM) algorithm for increasing the Overhauser magnetometer precision, and the precision is improved because of average calculation adopted. However, only rising edge of square wave is captured, the rest of rising edge and all falling edge of the square wave are missed. J. Ge [25] developed a multichannel interpolation frequency measurement method based on FPGA for Larmor frequency of the marine Overhauser sensor, the more channels there are, the higher the precision is. Yet the number of channels is limited by the resource of FPGA. An optimal multi-average frequency measurement is proposed in [26] to increase the frequency measurement precision. Nevertheless, only partial rising edge of square wave is used to reduce the trigger error. A linear model of frequency measurement method is presented in [27] to estimate the frequency of noised sine signal, the precision of frequency estimation is increased obviously because the Least Squares (LS) regression method is used to fit the linear curve. Nevertheless, the magnitude of the FID signal decays with an exponential curve. If the linear model is adopted directly, the feasibility must be verified.

\section{MODELLiNG}

\section{A. Modelling of frequency measurement for FID signal}

Assuming that the square wave ' $f_{x}$ ' shown in Fig.1.c) is converted from no noise sine signal by a hysteresis comparator, the following equation always holds if the amplitude of sine signal is invariable.

$$
T(i)=A \times i+B
$$

Where $i$ is the time of the rinsing edge of square wave. $T(i)$ is the time of the $i$-th rising edge of square wave. $A$ is the slope of the line 'L1' in Fig.1.c), which is the period of the frequency to be measured. $B$ is the time length from start point of frequency measurement to the first rising edge of square wave.

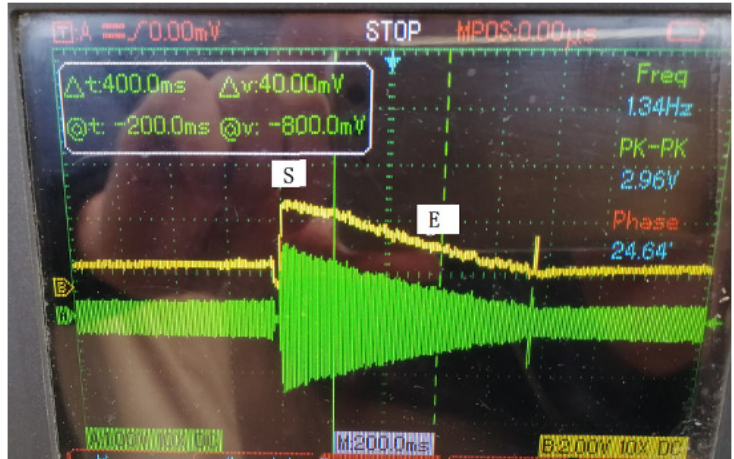

a)

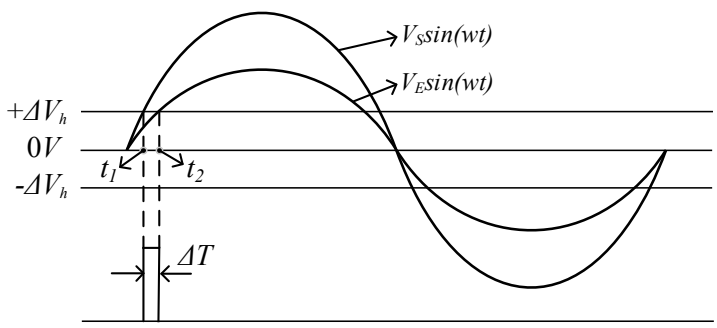

b)


c)

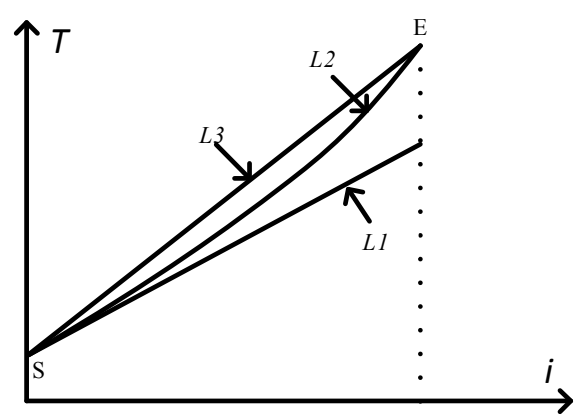

d)

Fig.1. The nonlinear modelling of frequency measurement for the FID signal, a) a wave picture of amplified FID signal, b) Trigger time deviation caused by the amplitude variations of the sine wave at the hysteresis comparator, c) the nonlinear curve of counting number for the FID signal, d) three curves, 'L1' is the straight line of counting number for the standard sine signal, 'L2' is the curve of counting number for FID signal, 'L3' is fitted straight line using two endpoint of curve 'L2'. 
As mentioned above, the amplitude of the amplified FID signal shown in Fig.1.a) decays with exponential curve, there must be the trigger time deviation because of different amplitudes of the FID signal, which is shown as Fig.1.b).

Assuming that the point ' $S$ ' in Fig.1.a) is the first waveform of the signal, and the point ' $\mathrm{E}$ ' is the last waveform, the relationship of the amplitude at two points can be expressed as

$$
V_{E}=V_{S} e^{-k t}
$$

Where $V_{A}$ is the amplitude of the point ' $\mathrm{S}$ '. $V_{B}$ is the amplitude of the point ' $\mathrm{E}$ '. $k$ is a constant, which is determined by the PM sensor and the gradient of the magnetic-field to be measured.

The trigger time deviation at point ' $\mathrm{B}$ ' can be deduced as follows. Assuming that the hysteresis voltage of the hysteresis comparator is $\Delta V_{h}$, then

$$
\begin{gathered}
V_{S} \sin \left(\omega t_{1}\right)=\Delta V_{h} \\
V_{E} \sin \left(\omega t_{2}\right)=\Delta V_{h}
\end{gathered}
$$

Where $\omega$ is the angular frequency of the frequency to be measured, $t_{1}$ and $t_{2}$ are the trigger times of the different amplitude sine waves, which is shown as Fig.1.b).

If $t_{1}$ and $t_{2}$ are small enough, expression (3) and (4) can be approximately rewritten as

$$
\begin{aligned}
& V_{S} \omega t_{1} \approx \Delta V_{h} \\
& V_{E} \omega t_{2} \approx \Delta V_{h}
\end{aligned}
$$

Then the trigger time deviation $\Delta T_{E}$ of the point ' $E$ ' can be expressed as

$$
\Delta T_{E}=t_{2}-t_{1}=\frac{\Delta V_{h}}{\omega}\left(\frac{1}{V_{E}}-\frac{1}{V_{S}}\right)=\frac{1}{V_{S}} \frac{\Delta V_{h}}{\omega}\left(e^{k T_{E}}-1\right)
$$

Where $T_{E}$ is the time from point ' $\mathrm{S}$ ' to point ' $\mathrm{E}$ '.

According to (7), the trigger time deviation $\Delta T_{i}$ can be directly written as

$$
\Delta T_{i}=\frac{1}{V_{S}} \frac{\Delta V_{h}}{\omega}\left(e^{k T_{i}}-1\right)
$$

Where $T_{i}$ is the time of the $i$-th waveform of the FID signal, which can be expressed as

$$
T_{i}=T \times i
$$

Then, (1) should be revised as

$$
\hat{T}(i)=A \times i+B+\Delta T_{i}=A \times i+B+\frac{T}{2 \pi} \frac{\Delta V_{h}}{V_{S}}\left(e^{k T i}-1\right)
$$

The curve 'L2' in Fig.1.d) can be used to express (10).

\section{B. Linearization and feasibility analysis}

According to (10) we know that, i) there are five unknown parameters in (10), they are $A, B, \Delta V_{h}, V_{S}$, and $k$. ii) the curve includes two parts, one is a straight line expressed as (1), another is an exponential curve expressed as (8). So, if we want to obtain the frequency of the FID signal, the parameter $A$ in (10) must be precisely solved by the analytic method.

The MCFM method was commonly used to measure the frequency if there was no noise in the sine signal. Only two endpoints shown as point ' $S$ ' and 'E' in Fig.1.a) and Fig.1.d) are used to calculate the measured frequency for the method, and the straight line is just like 'L3' in Fig.1.d). The slope of 'L1' and 'L3', respectively, can be expressed as,

$$
\begin{gathered}
A_{L 1}=\frac{T(N)-T(0)}{N-0}=A \\
A_{L 3}=\frac{\hat{T}(N)-\hat{T}(0)}{N-0}=A+\frac{1}{N} \frac{T}{2 \pi} \frac{\Delta V_{h}}{V_{S}}\left(e^{k T N}-1\right)
\end{gathered}
$$

Where $N$ is the number of the last waveform in Fig.1.c).

Then the deviation of the slope between two curves can be deduced as

$$
\Delta T=A_{L 3}-A_{L 1}=\frac{1}{N} \frac{T}{2 \pi} \frac{\Delta V_{h}}{V_{A}}\left(e^{k T N}-1\right)
$$

As mentioned above, the slope of the line is the period of the frequency to be measured. Therefore, the relative error of the measured frequency can be expressed as

$$
\delta=\frac{\Delta T}{T}=\frac{1}{N} \frac{1}{2 \pi} \frac{\Delta V_{h}}{V_{A}}\left(e^{k T N}-1\right)
$$

According to (14), the absolute error of the measured frequency is

$$
\Delta f_{N}=\frac{\Delta T}{T} f=\frac{1}{N} \frac{1}{2 \pi} \frac{\Delta V_{h}}{V_{A}}\left(e^{k N T}-1\right) f=\frac{1}{N T} \frac{1}{2 \pi} \frac{\Delta V_{h}}{V_{A}}\left(e^{k N T}-1\right)
$$

Equation (15) indicates that, if the MCFM method is used to measure the frequency of the FID signal, the absolute error of frequency measurement is $\Delta f_{N}$, moreover, the frequency value obtained by the MCFM method is smaller than the actual frequency.

Ordering

$$
M=\frac{V_{B}}{V_{A}}
$$


Where $M$ is the amplitude attenuation factor, which can be used to evaluate the quality of the FID signal. Compared with (2), $M$ can be expressed as

$$
M=1 / e^{k N T}
$$

The parameter $N T$ in (15) and (17) is the time duration of the measurement.

If the MCFM method is adopted to measure the frequency of the FID signal, there are three errors: error of standard frequency $\left(\Delta f_{s}\right)$, method error of $\operatorname{MCFM}\left(\Delta f_{M}\right)$, and error of trigger time $\left(\Delta f_{N}\right)$. They are uncorrelated random variables, and $\Delta f_{s}$ can be ignored if a high stability Oven Controlled Crystal Oscillator (OCXO) is adopted. So, the total synthesis error of the MCFM method can be expressed as

$$
\Delta f_{M C F M}=\sqrt{\Delta f_{N}^{2}+\Delta f_{M}^{2}}=\frac{1}{N T} \sqrt{\left(\frac{1}{2 \pi} \frac{\Delta V_{h}}{V_{A}}\left(e^{k N T}-1\right)\right)^{2}+\left(\frac{f_{x}}{f_{s}}\right)^{2}}
$$

Where $f_{s}$ is the standard frequency, $f_{x}$ is the frequency to be measured.

The frequency range of the FID signal is from $800 \mathrm{~Hz}$ to $5000 \mathrm{~Hz}$ in the range of the geomagnetic-field. If the value of the standard frequency is $100 \mathrm{MHz}$, and the time duration of the measurement is 1 second, correspondingly, the maximum value of $\Delta f_{M}$ is $5 \times 10^{-5} \mathrm{~Hz}$. However, $\Delta f_{N}$ equals $5.2 \times 10^{-4} \mathrm{~Hz}$ in spite of the signal quality being the best one ( $k$ in (18) equals 0.5 ), which is almost an order larger than the method error. So the nonlinear error is the main factor to reduce the measurement precision of the FID signal.

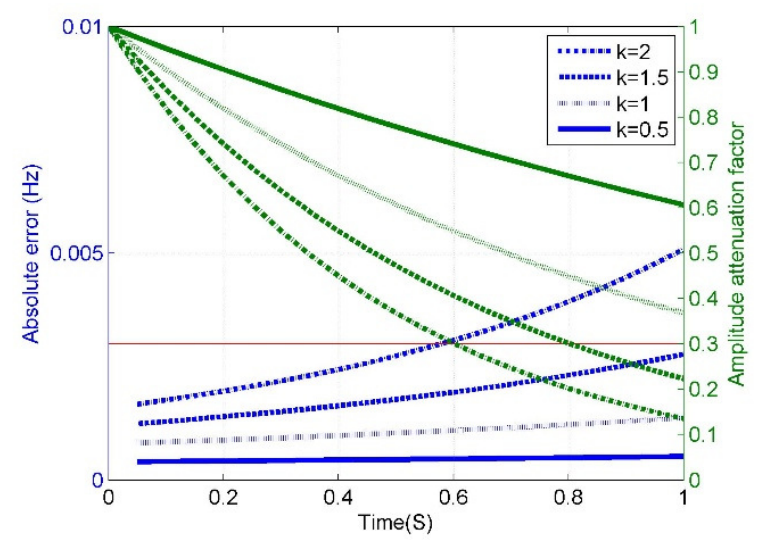

Fig.2. The curves of (17) and (18): the right y-axis is the curve of the FID signal with different $\mathrm{k}$, the left $\mathrm{y}$-axis is the curve of the maximum absolute error of the MCFM method.

Fig.2., which is drawn according to (17) and (18) supposing that $\Delta V$ and $\Delta V / V_{A}$ equals $5 \mathrm{mV}$ and 0.005 , respectively, can be used to evaluate the precision of frequency measurement. As shown, 1) the measurement error increases with the length of measurement time, the longer the measurement time is, the larger the measurement error is. This is contrary to the method error of the MCFM, which decreases with the time duration of measurement. 2) The measurement error decreases with the quality of the FID signal, the smaller the value of $k$ is, the better quality of the FID signal is, and the smaller measurement error is. 3) If the value of $k$ is smaller than one, the maximum measurement error is smaller than $3 \mathrm{mHz}$ in one second, correspondingly, absolute precision of $\mathrm{PM}$ is $3 \mathrm{mHz} \times 23.4874 \mathrm{nT} / \mathrm{Hz}$ according to [26], so it is better than $0.07 \mathrm{nT}$.

Above all, although the frequency measurement model of the FID signal is nonlinear, the precision of frequency measurement is still enough if the MCFM method is adopted to measure the frequency of no noise FID signal. So a linear model expressed as (19) can be used to replace the nonlinear model expressed as (10) on the following conditions: i) the quality of the FID signal is good enough, which can be evaluated by the envelope of the FID signal. Usually, if $k$ in (17) is smaller than one, the quality of the FID signal is considered as a good one. ii) There is no noise in the FID signal.

$$
T(i)=A_{L} \times i+B_{L}
$$

\section{REGRESSION ANALYSIS FOR LINEARIZATION MODEL}

\section{A. Least squares fitting}

The SNR of the amplified FID signal is very low because the maximum amplitude is about one microvolt, which has been discussed in [26] in detail. So, a trigger time error could be brought in the process of waveform transformation, which is shown as Fig.3. Then, (18) should be revised as

$$
\begin{aligned}
\Delta f_{M C F M} & =\sqrt{\Delta f_{N}{ }^{2}+\Delta f_{M}{ }^{2}+\Delta f_{R}^{2}} \\
& =\frac{1}{N T} \sqrt{\left(\frac{1}{2 \pi} \frac{\Delta V_{h}}{V_{A}}\left(e^{k N T}-1\right)\right)^{2}+\left(\frac{f_{x}}{f_{s}}\right)^{2}++\left(\frac{1}{\pi} \frac{1}{S N R P}\right)^{2}}
\end{aligned}
$$

Where $\Delta f_{R}$ is the error caused by additional random noise, and SNRP stands for the peak signal-noise-ratio, which has been discussed in [24] in detail. Usually, the value of SNRP decays from $50 \mathrm{~dB}$ to $30 \mathrm{~dB}$ if the quality of the FID signal is good enough, correspondingly, $\Delta f_{R}$ is about $10 \mathrm{mHz}$. Compared with other errors in (20), the error of frequency measurement caused by trigger time error is the main reason to reduce the measurement precision of PM. So, the total error can be approximately expressed as

$$
\Delta f_{M C F M} \approx \Delta f_{R}=\frac{1}{N T} \frac{1}{\pi} \frac{1}{S N R P}
$$

If the Least Squares (LS) method is used to fit the linearization model discussed in Section 2 there are two benefits for the frequency measurement of the FID signal: 
i) Trigger time error will be greatly reduced because the noise is an additional random noise, which indicates that the value of trigger error $\Delta f_{R}$ will greatly decrease if the LS method is adopted.

ii) The position of the fitted straight line, which is shown as ' $L$ ' in Fig.4., should be between the straight line 'L1' and 'L3', which indicate that the value of nonlinear error $\Delta f_{N}$ will be further reduced if the LS method is adopted.

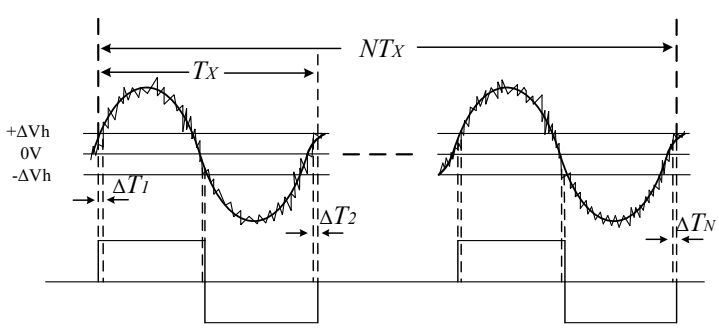

a)

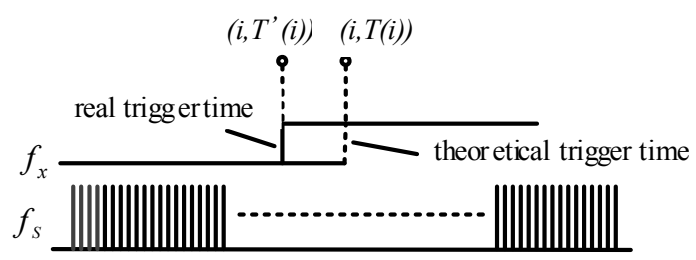

b)

Fig.3. The trigger error, a) trigger time error caused by random noise, $b$ ) the counting error caused by trigger time error.

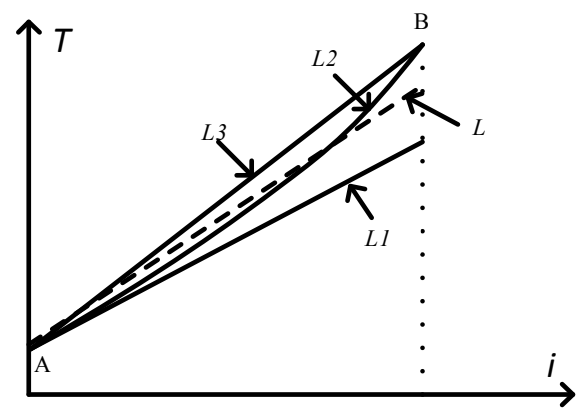

Fig.4. The positional relationship of four curves, 'L1' is the straight line of no noised sine signal, 'L2' is the curve of the FID signal, 'L3' is the straight line using the MCFM method, 'L4' is the fitted straight line using the LS method.

Assuming that $T^{\prime}(i)$ is the counting number of the standard frequency at the $i$-th rising edge of the frequency to be measured, the slope of (19) can be deduced by the LS method [27], and can be expressed as

$$
A_{L}=\frac{N \sum_{i=0}^{N} i T^{\prime}(i)-\sum_{i=0}^{N} i \sum_{i=0}^{N} T^{\prime}(i)}{N \sum_{i=0}^{N} i^{2}-\left(\sum_{i=0}^{N} i\right)^{2}} \times T_{s}
$$

Then, the measured frequency $f_{x}$ can be deduced as

$$
f_{x}=\frac{1}{A_{L}}=\frac{N \sum_{i=0}^{N} i^{2}-\left(\sum_{i=0}^{N} i\right)^{2}}{N \sum_{i=0}^{N} i T^{\prime}(i)-\sum_{i=0}^{N} i \sum_{i=0}^{N} T^{\prime}(i)} \times f_{s}
$$

\section{B. Error analysis}

There are two errors if the LS method is adopted to fit the linearization model. The first one is the method error, which is caused by the random error, and has been discussed in [27]. So it can be written as

$$
\Delta f_{(M, L S)}=\frac{2 \sqrt{3}}{N T \sqrt{N-2}} \frac{1}{\pi} \frac{1}{S N R P}
$$

The second one is the nonlinear error caused by the LS method, and which can be expressed as

$$
\Delta f_{(N, L S)}=\frac{A_{L}-A}{A} f_{s}
$$

Then, the total error of the proposed method can be expressed as

$$
\Delta f_{L S}=\sqrt{\Delta f_{(M, L S)}^{2}+\Delta f_{(N, L S)}^{2}}
$$

Assuming that the time duration of measurement is larger than 0.6 seconds, and the counting number is larger than 1000 , correspondingly, the method error in (24) is smaller than 2 $\mathrm{mHz}$. Except that, $A_{L}$ should be smaller than $A_{L 3}$, so the nonlinear error caused by the LS method should be smaller than $5.2 \times 10^{-4} \mathrm{~Hz}$ according to Section 2.2. In a word, compared with the error caused by random noise, the nonlinear error can be ignored, so the total error of the proposed method can be approximately expressed as

$$
\Delta f_{L S} \approx \Delta f_{(M, L S)}=\frac{2 \sqrt{3}}{N T \sqrt{N-2}} \frac{1}{\pi} \frac{1}{S N R P}
$$

Compared with (21), the error of the proposed method is about $2 \sqrt{3} / \sqrt{N-2}$ times smaller than the MCFM method for the frequency measurement of the FID signal, and is about $2 \sqrt{30} / \sqrt{N-2}$ times smaller than the MPM method described in [24].

\section{Method implementation}

The capture function of timer in STM32 can be used to obtain the raw data to implement the proposed method, which is shown as Fig.5. The input capture channel 1 of the TIM2 in STM32F207 is set as input rising edge capture mode. After 
the timer is started, whenever a rising edge appears at the PA1 pin, the counting number of TIM2 will be automatically loaded into the capture register of channel 1 by the STM32. The captured number is then stored in the array $a[i]$ in the interrupt service subroutine, which is displayed in Fig.5. If all of $a[i]$ are filled with the captured values, $T^{\prime}(i)$ in (23) can be expressed as

$$
T^{\prime}(i)=a[i]
$$

Substituting (28) into (23), the estimated value of $f_{x}$ can be calculated.

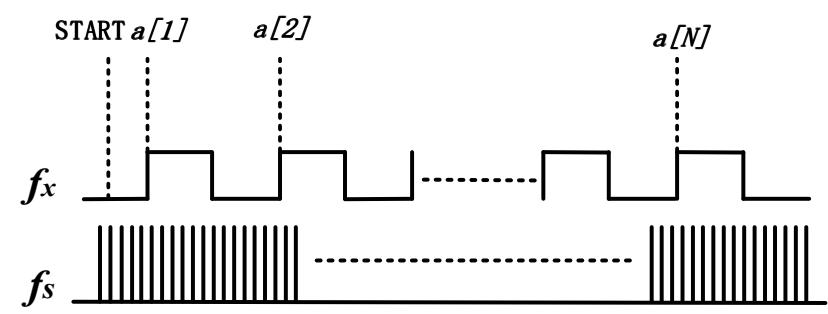

Fig.5. Raw data captured method by STM32.

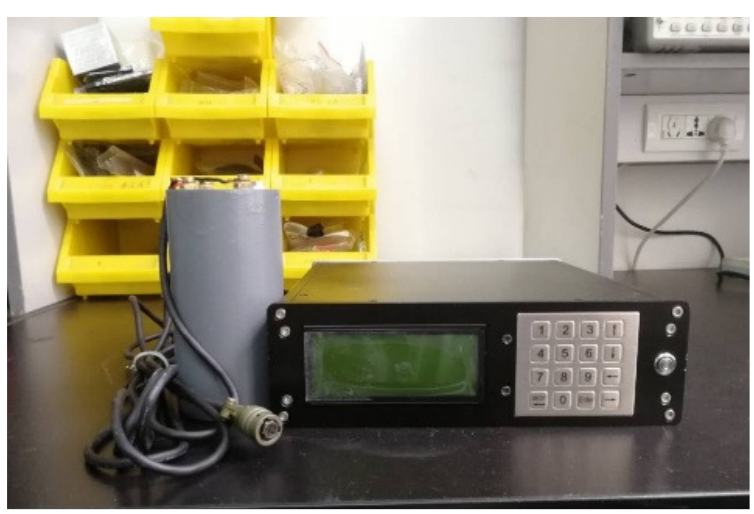

a)

\section{EXPERIMENT AND ANALYSIS}

\section{A. PM Prototype}

A PM prototype shown as Fig.6.a) and Fig.6.b), consisting of a PM sensor, a digital board, an analog board, a shell, and a battery fixed in shell, was developed to verify the effectiveness of the proposed method for improving the precision and sensitivity of PM. Combined with a signal generator (33500B, keysight), the developed test platform is displayed in Fig.6.d). The signal generator is used for the indoor frequency measurement comparison experiment. The PM sensor, the structure of which is shown as Fig.6.c), is used for the outdoor magnetic-field measurement experiment. The structure and the working principle of the PM sensor has been discussed in [28] in detail.

The amplifier in the analog board is used to amplify the weak FID signal, the output of which forms the PM sensor, the gain of the amplifier is about $200,000 \mathrm{~V} / \mathrm{V}$. A series resonant circuit, which is composed of two inductors in the PM sensor and a variable capacitor bank in the analog board, is designed to pre-amplify the FID signal. Meanwhile the Q value of the series resonant circuit is about 10 . Consequently, the FID signal output from the PM sensor is amplified about 2,000,000 times, and the amplitude of the amplified FID signal is about $2 \mathrm{Vpp}$.



b)

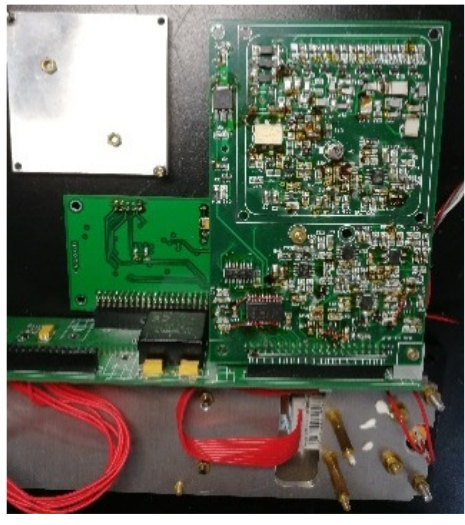

c)

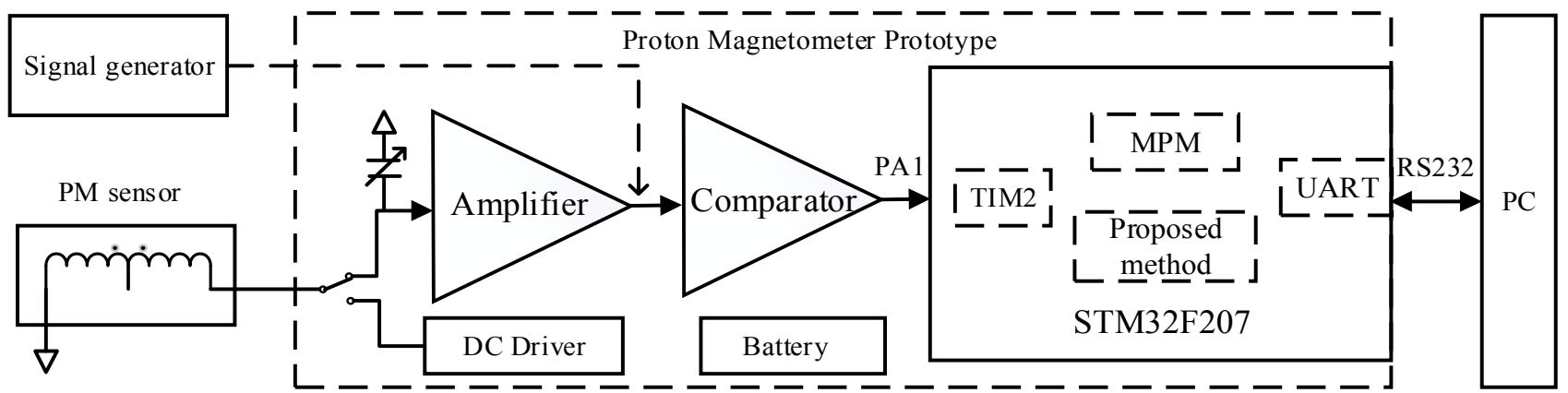

d)

Fig.6. Test platform, a) PM prototype with a PM sensor, b) the circuit of the PM prototype, c) the structure of PM sensor, d) the structure of the test platform including signal generator, the PM sensor and the PM prototype. 


\section{B. Indoor experiment}

Two indoor experiments were performed to verify the effectiveness of the proposed method to deal with the noised sine signal. The length of measurement time of both methods was set to 800 milliseconds for the indoor experiment.

The first experiment was the contrast experiment of frequency measurement accuracy at a fixed SNR. The frequency range was set from $800 \mathrm{~Hz}$ to $5000 \mathrm{~Hz}$, which corresponds with the global geomagnetic-field range. A noised sine signal was generated by a signal generator as the frequency to be measured. The amplitude of the sine signal was set to $2 \mathrm{Vpp}$, and the frequency was varied from $800 \mathrm{~Hz}$ to $5000 \mathrm{~Hz}$. Moreover, the random noise, which is generated by modulation function of the signal generator, was added to the sine signal. The bandwidth of noise was set to $20 \mathrm{MHz}$, and the amplitude percentage of noise-to-signal (APNS) was set to $15 \%$ or $30 \%$. Hence, the peak-to-peak SNR of the generated signal was about $20.7 \mathrm{~dB}$ if the APNS was set to $30 \%$ (the peak coefficient of random noise is 4.6 ), and about $26.7 \mathrm{~dB}$ if the APNS was set to $15 \%$. When the experiment was performed, the generated signal was provided as the input signal of comparator directly, which is shown as Fig.6.d).

Fig.7. is the frequency measurement result of two methods when the frequency is $1900 \mathrm{~Hz}$. The X-axis in Fig.7.a) and Fig.7.c) are the measurement times, and the $\mathrm{Y}$-axis stand for

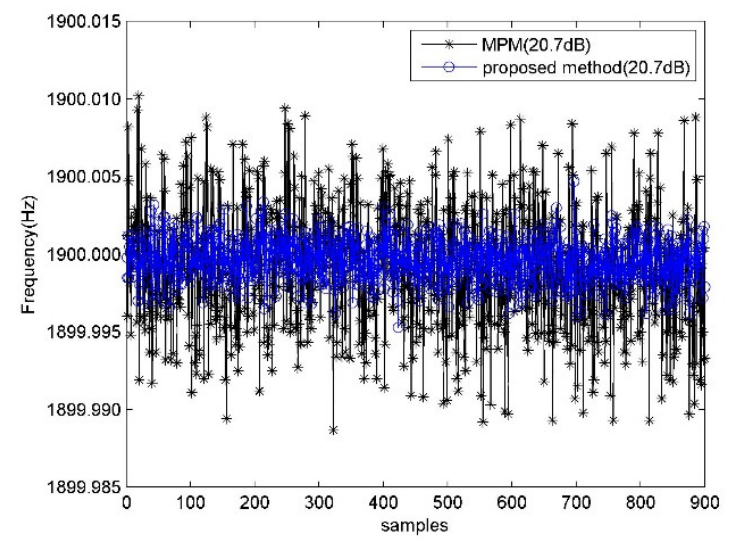

a)

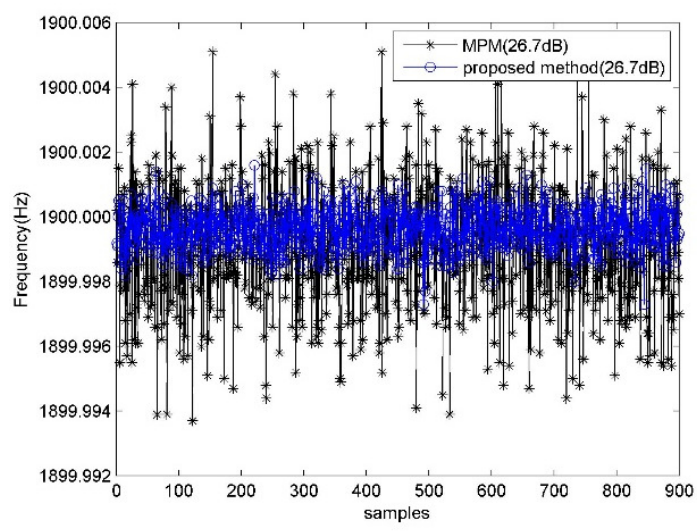

c) the result of each time of frequency measurement. From Fig.7. we can see that:

i) No matter what the SNR is, the probability density distribution of the proposed method is better than the MPM method.

ii) The higher SNR is, the narrower the width of the probability density distribution curve is, and the larger peak value of the probability density distribution is.

iii) Except that, when the SNR of the signal to be measured is $20.7 \mathrm{~dB}$ (the curve is shown as Fig.7.a)), the Root-MeanSquare Deviation (RMSD) of the proposed method and the MPM method is $1.3 \mathrm{mHz}$ and $7.9 \mathrm{mHz}$, respectively, the average value of the frequency measured by two methods is $1899.9996 \mathrm{~Hz}$ and $1899.9989 \mathrm{~Hz}$, respectively, and the maximum error is $5.1 \mathrm{mHz}$ and $11.2 \mathrm{mHz}$. When the SNR equals $26.7 \mathrm{~dB}$ (the curve is shown as Fig.7.c)), the RMSD is $0.6 \mathrm{mHz}$ and $1.9 \mathrm{mHz}$, respectively, the average value is $1899.9996 \mathrm{~Hz}$ and $1899.9991 \mathrm{~Hz}$, respectively, and the maximum error is $2.3 \mathrm{mHz}$ and $6 \mathrm{mHz}$. If the proposed method is adopted by PM, the maximum absolute error of magnetic-field measurement is about $0.12 \mathrm{nT}$ when the SNR is $20.7 \mathrm{~dB}$, and $0.05 \mathrm{nT}$ when the SNR is $26.7 \mathrm{~dB}$. From the calculation result we can see that, if the SNR of the FID signal is larger than $26.7 \mathrm{~dB}$, the absolute precision of PM could be better than $0.1 \mathrm{nT}$.

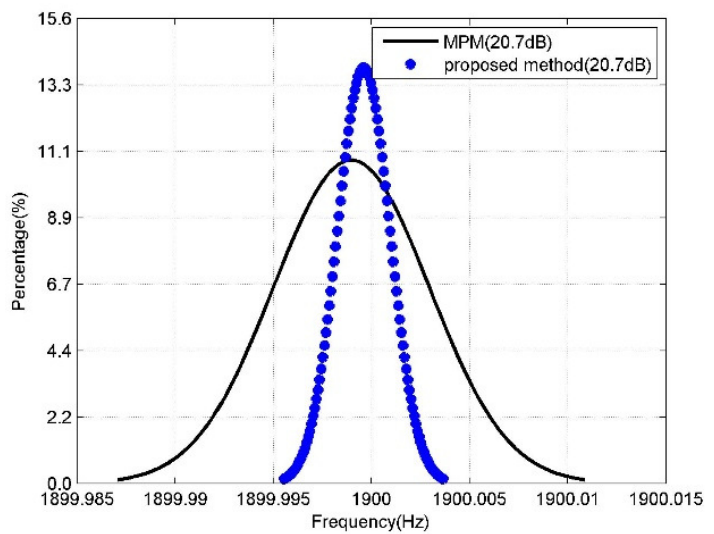

b)

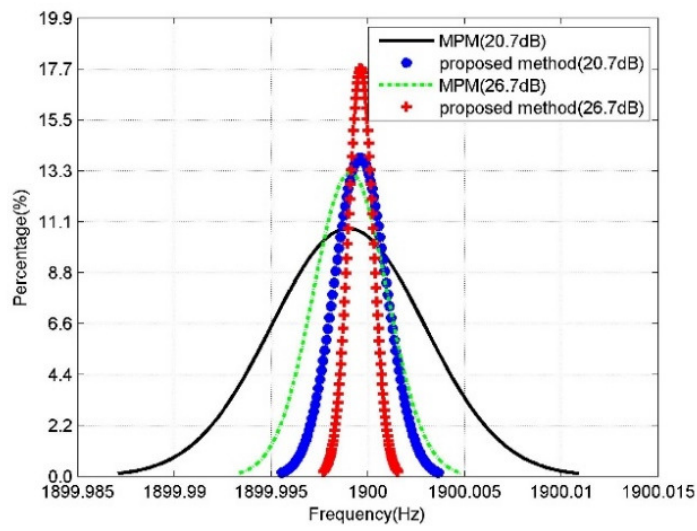

d)

Fig.7. Experimental result of frequency measurement of low SNR sine signal, the frequency is $1900 \mathrm{~Hz}$, a) the value of frequency measured by two methods when the SNR is $20.7 \mathrm{~dB}, \mathrm{~b}$ ) the probability density distribution map of a), c) the value of frequency measured by two methods when the SNR is $26.7 \mathrm{~dB}$, d) the probability density distribution map of a) and c). 
Table 1. Comparing result of different methods, $S N R=20.7 \mathrm{~dB}$, frequency $=1900 \mathrm{~Hz}$.

\begin{tabular}{|l|l|l|l|l|l|l|l|}
\hline method & Performed mode & $\begin{array}{l}\text { Sample } \\
\text { method }\end{array}$ & $\begin{array}{l}\text { Sample } \\
\text { rate }\end{array}$ & $\begin{array}{l}\text { Sample } \\
\text { length }\end{array}$ & $\begin{array}{l}\text { Time length } \\
\text { of sampling }\end{array}$ & $\begin{array}{l}\text { Executing } \\
\text { time }\end{array}$ & RMSD \\
\hline Interpolation FFT & simulation (PC, matlab) & ADC & $10 \mathrm{~K} \mathrm{sps}$ & 2048 & $\sim 200 \mathrm{~ms}$ & $\sim 1 \mathrm{~s}$ & $25 \mathrm{mHz}$ \\
\hline Prony & simulation (PC, matlab) & ADC & $10 \mathrm{~K} \mathrm{sps}$ & 2048 & $\sim 200 \mathrm{~ms}$ & $>20 \mathrm{~s}$ & $3.1 \mathrm{mHz}$ \\
\hline SVD & simulation (PC, matlab) & ADC & $10 \mathrm{~K} \mathrm{sps}$ & 2048 & $\sim 200 \mathrm{~ms}$ & $\sim 10 \mathrm{~s}$ & $2.7 \mathrm{mHz}$ \\
\hline MPM & experiment (prototype, C) & CMP+CNT & $1.9 \mathrm{~K} \mathrm{sps}$ & $\sim 2000$ & $\sim 1.0 \mathrm{~s}$ & $\sim 1 \mathrm{~ms}$ & $7.9 \mathrm{mHz}$ \\
\hline Proposed method & experiment (prototype, C) & CMP+CNT & $1.9 \mathrm{~K} \mathrm{sps}$ & $\sim 2000$ & $\sim 1.0 \mathrm{~s}$ & $\sim 10 \mathrm{~ms}$ & $1.3 \mathrm{mHz}$ \\
\hline
\end{tabular}

The second contrast experiment is the frequency measurement accuracy with different SNR at a fixed frequency. The frequency and the amplitude of the measured sine signal were set to $1900 \mathrm{~Hz}$ and $2 \mathrm{Vpp}$, respectively. The random noise, which is generated by modulation function of the signal generator, was added to the sine signal. The bandwidth of noise was set to $20 \mathrm{MHz}$, and the amplitude percentage of noise-to-signal was varied from $1 \%$ to $30 \%$. Correspondingly, the SNR of the noised sine signal was varied from $50.2 \mathrm{~dB}$ to $20.7 \mathrm{~dB}$. The RMSD of two methods for different SNR sine signals are shown in Fig.8., and each RMSD is calculated by 200 samples. According to the figure we can see that the RMSD of the proposed method was about 5 times smaller than the MPM method when the SNR of measured sine signal is varied from $20 \mathrm{~dB}$ to $50 \mathrm{~dB}$.

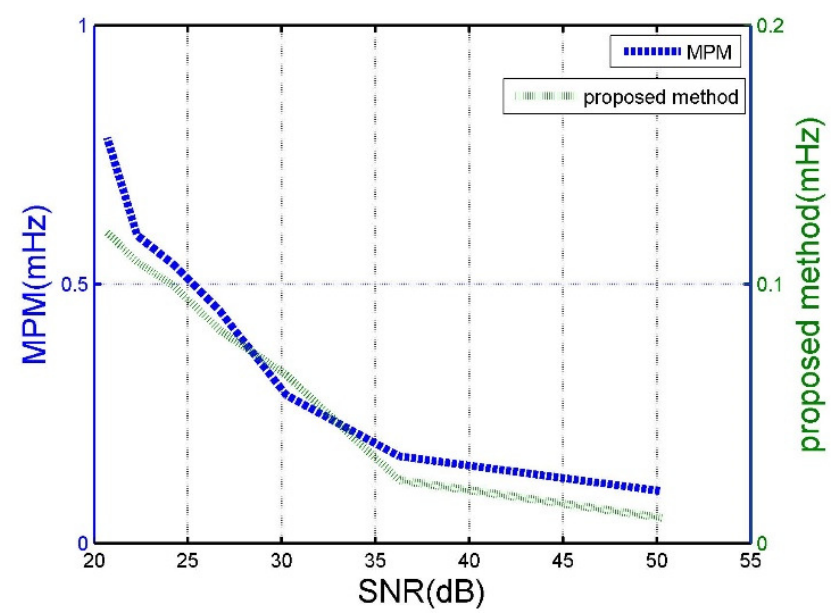

Fig.8. The RMSD of two methods for different SNR sine signal.

According to the experiments above, comparison results of five frequency estimation methods for noised sine signal: Interpolation FFT, Prony, SVD, MPM, and the proposed method, are listed in Table 1. CMP in Table 1. stands for comparator, and CNT stands for counter.

Form the results in Table 1., the time duration of sampling of previous three methods is smaller than that of the latter two methods, nevertheless, the executing time is much longer than that of the latter two methods. Moreover, the RMSD of the proposed method is the best one compared with other methods.

\section{Outdoor experiment}

Outdoor experiment was performed to verify the effectiveness of the proposed method to increasing the PM precision compared with the MPM method. The experiment field is shown as Fig.9.a). The PM sensor is located far away from the PM prototype to reduce the electromagnetic interference. The sampling time interval was set to two seconds, one second was used to active the PM sensor, and the rest time was used to measure the frequency, display magnetic-field value on an LCD and send it to the PC. Once captured waveform of the measurement process is shown as Fig.9.b).

Fig.9.c) is the curve drawn by 800 sampled data, which indicate that the curve of the proposed method is smoother than that of the MPM method, and the geomagnetic-field varies slowly during a long period of time. Fig.9.d) is the curve drawn by 100 samples selected from Fig.9.c), which indicate that the geomagnetic-field is almost not variable in a short time. Moreover, the RMSD of the two methods is $0.13 \mathrm{nT}$ and $0.32 \mathrm{nT}$, respectively for the data of Fig.9.d). Fig.9.e) and Fig.9.f) are the Noise Power Spectral Density (NPSD) of the measured magnetic-field by two methods. From Fig.9.f) we can see that the NPSD of the proposed method is about $80 \mathrm{pT} / \mathrm{Hz}^{1 / 2}$ if the frequency is larger than $0.1 \mathrm{~Hz}$. Nevertheless, the NPSD of the MPM method is about $110 \mathrm{pT} / \mathrm{Hz}^{1 / 2}$ at the same frequency range. The results discussed above indicate that the proposed method can be used to increase the measurement precision of PM.

Moreover, the actual measurement precision of the PM prototype is smaller than the result of frequency measurement discussed in Section 4, B. The result could be caused by two reasons: the first one is that the geomagnetic-field is variable in time, and the second one is that the extra noise interfaces with the coils in the PM sensor and the connection cable between sensor and prototype. 




a)

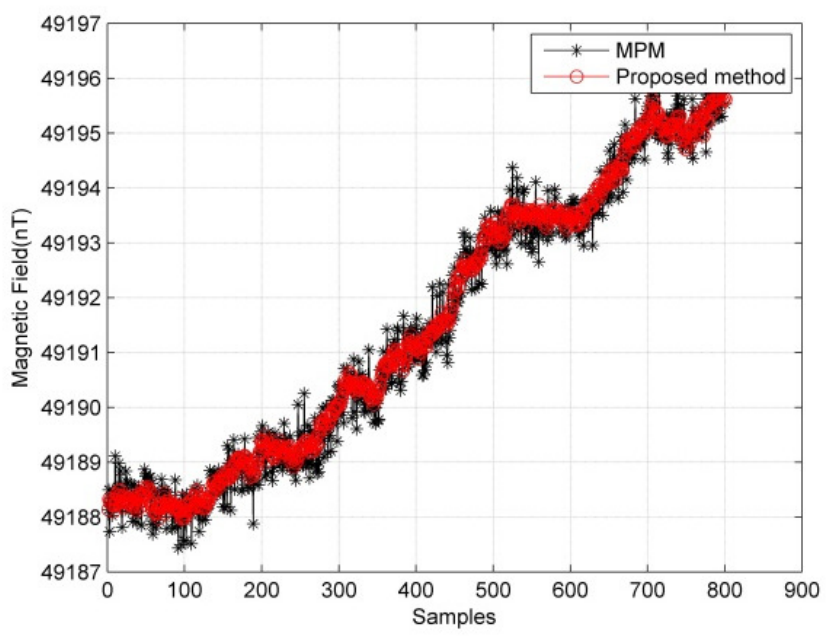

c)



e)

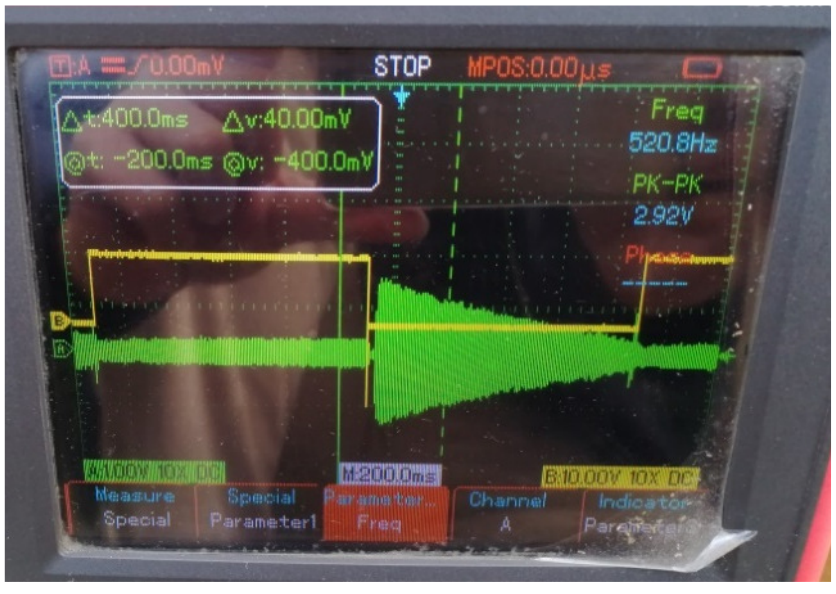

b)

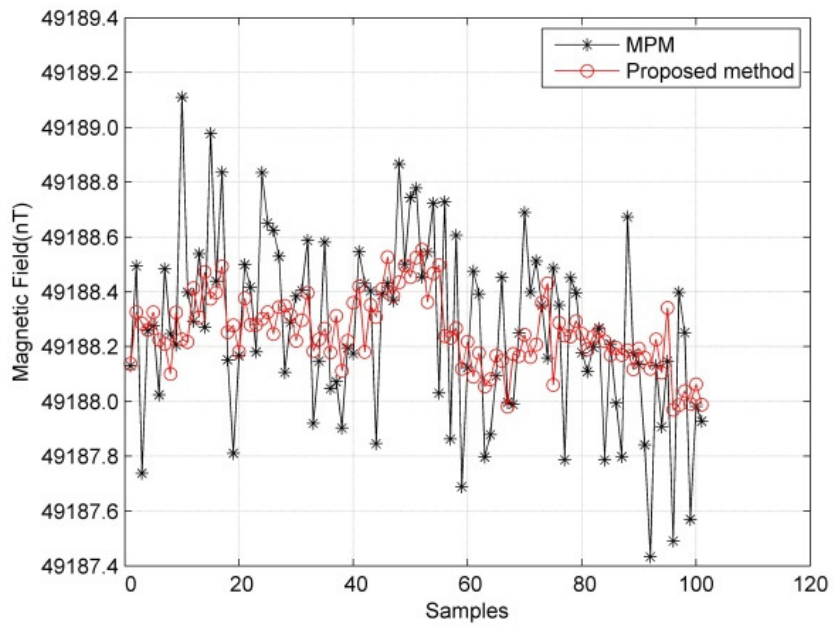

d)



f)

Fig.9. Outdoor experiment result, a) experimental field, b) captured waveform of one-time measurement, c) the curve of measured geomagnetic-field, d) 100 samples selected form a), e) the NPSD curve of c), f) the linear fitted curve of the NPSD when the frequency is larger than $0.09 \mathrm{~Hz}$. 


\section{CONCLUSION}

This paper presents a nonlinear model of frequency measurement for the FID signal, a linearized method for the nonlinear model under limited conditions, and a frequency estimation method for the linearized model. The experimental results have validated the proposed method for increasing the precision of noised sine signal and the FID signal, demonstrating its practical feasibility. Moreover, the proposed method takes a very short time, and the implementation method is very easy. So the proposed method could be used in a similar application, such as the Overhauser Magnetometer.

\section{REFERENCES}

[1] Liu, H., Wang, H.P., Bin, J.C., Dong, H.B., Ge, J., Liu, Z., Yuan, Z.W., Zhu, J., Luan, X.Q. (2020). Efficient noise reduction for the free induction decay signal from a proton precession magnetometer with time-frequency peak filtering. Review of Scientific Instruments, 91 (4), 045101.

[2] Duret, D., Bonzom, J., Brochier, M., Frances, M., Leger, J.M., Odru, R., Salvi, C., Thomas, T., Perret, A. (1995). Overhauser magnetometer for the danish oersted satellite. IEEE Transactions on Magnetics, 31 (6), 3197-3199.

[3] Sapunov, V., Rasson, J., Denisov, A., Saveliev, D., Kiselev, S., Denisova, O., Podmogov, Y., Khomutov, S. (2001). Theodolite-borne vector overhauser magnetometer: Dimover. Earth Planets \& Space, 58 (6), 711-716.

[4] Shim, J.H., Lee, S.J., Hwang, S., Yu, K., Kim, K. (2015). Proton spin-echo magnetometer: A novel approach for magnetic field measurement in residual field gradient. Metrologia, 52 (4). 496-501.

[5] Acker, F.E. (1971). Calculation of the signal voltage induced in a toroidal proton-precession magnetometer sensor. IEEE Transactions on Geoscience Electronics, 9 (2), 98-103.

[6] Ge, J., Dong, H., Liu, H., Yuan, Z., Dong, H., Zhao, Z., Liu, Y., Zhu, J., Zhang, H. (2016). Overhauser geomagnetic sensor based on the dynamic nuclear polarization effect for magnetic prospecting. Sensors, $16(6), 806$.

[7] Tan, C., Dong, H., Ge, Z. (2010). Overhauser magnetometer excitation and receiving system design. Chinese Journal of Scientific Instrument, 31 (8), 18671872.

[8] Liu, H., Dong, H., Liu, Z. Ge, J. (2017). Noise characterization for the FID signal from proton precession magnetometer. Journal of Instrumentation, 12 (7), 07019-07019.

[9] Quinn, B.G. (1997). Estimation of frequency, amplitude, and phase from the DFT of a time series. IEEE Transactions on Signal Processing, 45 (3), 814817.

[10] Candan, C. (2011). A method for fine resolution frequency estimation from three $\mathrm{dft}$ samples. IEEE Signal Processing Letters, 19 (6), 351-354.
[11] Yamada, T. (2013). High-accuracy estimations of frequency, amplitude, and phase with a modified DFT for asynchronous sampling. IEEE Transactions on Instrumentation and Measurement, 62 (6), 1428-1435.

[12] Zhan, L., Liu, Y., Culliss, J., Zhao, J., Liu, Y. (2015). Dynamic single-phase synchronized phase and frequency estimation at the distribution level. IEEE Transactions on Smart Grid, 6 (4), 2013-2022.

[13] Shen, T., Li, H., Zhang, Q., Li, M. (2017). A novel adaptive frequency estimation algorithm based on interpolation FFT and improved adaptive notch filter. Measurement Science Review, 17 (1), 48-52.

[14] Kirianaki, N.V., Yurish, S.Y., Shpak., N.O. (2001). Methods of dependent count for frequency measurements. Measurement, 29 (1), 31-50.

[15] Wang, Z., Pan, L., Ji, G. (2013). Frequency measurement method utilizing discrete phase delay detection. Chinese Journal of Scientific Instrument, 34 (5), 994-1000.

[16] Balázs, R, István, K, Tamás, D. (2016). Efficient implementation of least squares sine fitting algorithms. IEEE Transactions on Instrumentation and Measurement, 65 (12), 2717-2724.

[17] Händel, P. (2010). Amplitude estimation using IEEESTD-1057 three-parameter sine wave fit: Statistical distribution, bias and variance. Measurement, 43 (6), 766-770.

[18] Bilau, T.Z., Megyeri, T., Sárhegyi, A., Márkus, J., Kollár, I. (2004). Four-parameter fitting of sine wave testing result: Iteration and convergence. Computer Standards \& Interfaces, 26 (1), 51-56.

[19] Xu, X., Wang, G., Wang, Y., Zhang, H., Li, X. (2009) Study on precise frequency measurement based on sound card. In 9th International Conference on Electronic Measurement \& Instruments. IEEE, 445448.

[20] Wang, H., Dong, H., Li, Z. (2015). The study of FID signal detection technology of proton magnetometer based on prony method. Journal of Information \& Computational Science, 9 (11), 3265-3272.

[21] Wang, H., Dong, H., Hao, G. Luo, Y., Yu, Z. (2012). The study of fid signal processing algorithm of proton magnetometer based on SVD. International Journal of Digital Content Technology \& Its Applications, 6 (18), 139-147.

[22] Wang, H., Dong, H., He, L., Meng, Q. (2012). The study of matrix mathematical model construction of PPM FID signal associate with FDM. Journal of Convergence Information Technology, 7 (16), 169-178.

[23] Nakao, T., Furukawa, T., Suematsu, T., Utsumi, H., Kubota, H. (2014). Intensity estimation method of the FID signal based on the high-order Prony Method including evaluation used to the priori information in quantitative NMR. In IEEE International Symposium on Signal Processing and Information Technology, 37 (574), 000067-000072. 
[24] Dong, H., Liu, H., Ge, J., Yuan, Z., Zhao, Z. (2016). A high-precision frequency measurement algorithm for FID signal of proton magnetometer. IEEE Transactions on Instrumentation \& Measurement, 65 (4), 898-904.

[25] Ge, J., Qiu, X., Dong, H., Luo, W., Liu, H., Yuan, Z., Zhu, J., Zhang, H. (2018). Short-time and highprecision measurement method for larmor frequency of marine overhauser sensor. IEEE Sensors Journal, 18 (4), 1442-1448.

[26] Tan, C., Wang, J., Li. Z. (2019). A frequency measurement method based on optimal multi-average for increasing proton magnetometer measurement precision. Measurement, 135, 418-423.
[27] Tan, C., Yue, Z., Wang, J. (2019). Frequency measurement approach based on linear model for increasing low SNR sinusoidal signal frequency measurement precision. IET Science, Measurement \& Technology, 13 (9), 1268-1276.

[28] Tan, C., Wang, J., Li, Z. (2018). Structural parameters design of square coil for proton magnetic field sensor. Chinese Journal of Sensors and Actuators, 31 (11) 1679-1683.

Received January 06, 2020

Accepted June 10, 2020 